\title{
Improving The Quality of The Line of Sight Angle Coordinates Filter of The Self- Guidance Missile When Using The Snake Style Maneuvering Model
}

\author{
Nguyen Van Bang ${ }^{1}$; Dang Cong Vu' ${ }^{2}$ Vu Quang Luong ${ }^{3}$ \\ Air Defence - Air Force Academy, Ha Noi, Viet Nam ${ }^{1,2,3}$ \\ Banghvpkkq@gmail.com ${ }^{1}$; dcongvu1981@gmail.com ${ }^{2}$; vuquangluong88@gmail.com ${ }^{3}$
}

DOI: 10.26821/IJSHRE.9.4.2021.9406

\begin{abstract}
On the basis of the tracking multi-loop target angle coordinate system of self-guidance missiles, the article presents the results of synthesis of line of sight angle coordinate filter when using the snake style maneuver model on the basis of applying the algorithm interactive multi-model adaptive filter to improve, advance the quality of the target phase coordinate filter. In addition to choosing 3 models to design the line of sight angle coordinate filter; The velocity model is constant, the Singer model and the acceleration model are constant, characteristic for 3 different levels of the target's maneuverability. The article also adds a layer of maneuvering target model type snake (maneuver form usually happens in practice) in during the synthesis of target phase coordinate filter. Because, a snake style maneuverable target is a complex form of maneuvering that the line of sight angle coordinate filter has absolutely no a priori information about the maneuvering type, the maneuvering amplitude and the maneuvering frequency. Simple algorithm, small evaluation error, fast and stable convergence, reliable simulation results.
\end{abstract}

Keywords: The snake style maneuvering, Angle of line of sight, Interactive multi-model, Kalman filter, Target.

\section{INTRODUCTION}

The tracking single-loop target angle coordinate system is used in today's flying equipment, although its structure is simple, but its accuracy is not high, especially in the case of maneuvering targets [1]. Therefore, the issue of synthesizing the coordinate determination system of the target angle with high precision is a practical problem that needs to be solved.

In the tracking multi-loop optimal target angle coordinate system [2], the maneuverability of the target is taken into account by the target normal acceleration model $\left(j_{t}\right)$. This model is characterized by maneuvering frequency $\left(\alpha_{j_{t}}\right)$ and maneuvering intensity $\left(\sigma_{j_{t}}^{2}\right)$. In this angular coordinate system, accuracy is enhanced by not using directly the signal equalization direction as the signal for evaluating the target coordinates, but using a separate line of sight angle coordinate evaluation filter [3]; at the same time the related states are evaluated by the Kalman filter algorithm in the tracking loop [4], [5]. However, since we can not choose a target model suitable for all types of maneuverability, the optimal angular coordinate system with fixed parameters will appear large evaluation errors, when in reality, meneuver targets is different from the selected model.

There are many adaptive filtering techniques that can be applied to tracking to maneuvering targets, but with multi-model filtering technique, it avoids solving the maneuver moment detection problem. For interactive multi-model filtering algorithm, just 
ISSN-2347-4890

Volume 9 Issue 4 April 2021

use $\mathrm{N}$ filters corresponding to $\mathrm{N}$ different maneuver models of the target [6], [7].

The tracking multi-loop target angle coordinate determination system, in which the line of sight angle coordinate filter uses the interactive multimodel (IMM) filter algorithm will significantly reduce the tracking error when the target changes type maneuver, including both an uncertainty related to maneuvering moment and maneuvering intensity. Because, the interactive multi-model evaluation algorithm is capable of adapting to maneuverability of the target as the evaluation process progresses to the most suitable model [8].

On the basis of the tracking multi-loop target angle coordinate system, 3 models selected to design the line of sight angle coordinate filter are constant velocity (CV) model, Singer model and constant acceleration (CA) model. However, the snake style maneuvering target model layer (the maneuvering type that often occurs in practice) is not taken into account during the synthesis of the target phase coordinate filter. The snake style maneuvering

target is a complex form of maneuvering where the line of sight angle coordinate filter has absolutely no prior information on the maneuvering type, maneuvering amplitude and maneuvering frequency. So, need to use the snake type maneuvering model layer when synthesizing the IMM evaluation algorithm to improve the quality of the line of sight angle coordinate filter.

\section{SYNTHESIS OF LINE OF SIGHT ANGLE COORDINATE FILTER}

If we have more a priori information about the maneuvering type of the target in reality, we can completely use the layer of snake style maneuvering models (temporarily called Weaving models) to synthesize the IMM filter algorithm, then the quality of the target angle coordinate system will be significantly improved.

To build the kinetics of the snake style maneuvering model, we need to expand the state space add a component that is the derivative of the target normal acceleration. The proposed model kinetics are as follows:
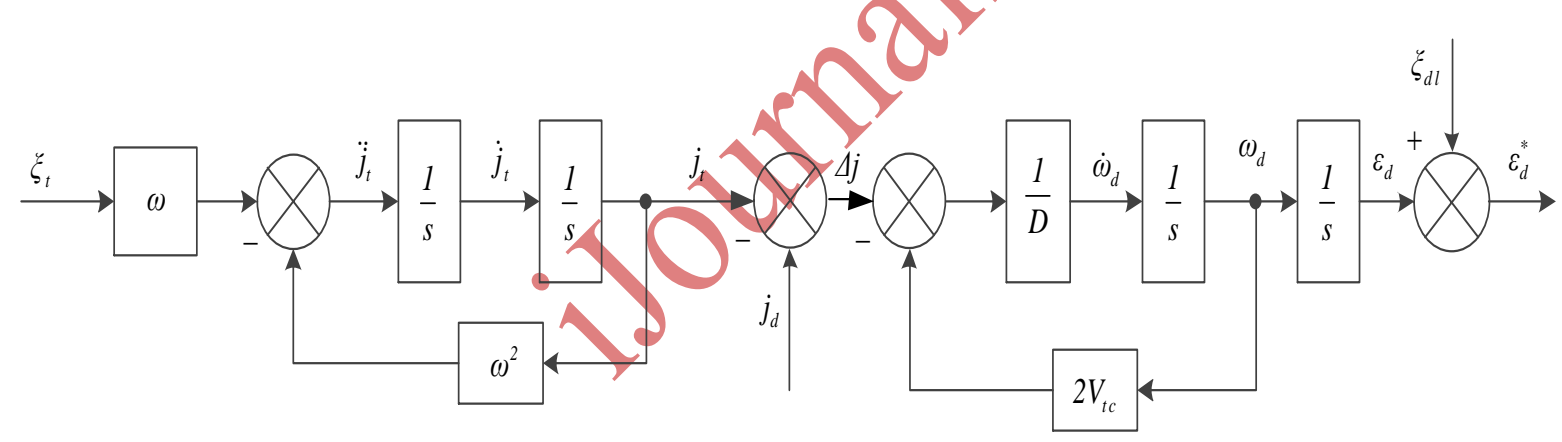

Figure 1. Weaving model for synthesis of $4^{\text {th }}$ Kalman filter

$D$ - Relative distance between missile and target;

$j_{t}$ - Normal acceleration of the target;

$j_{d}$ - Normal acceleration of the missile;

$\varepsilon_{d}$ - Angle of line of sight between missile and target;

$\omega_{d}$ - Speed of line of sight rotation;

$\xi_{j_{t}}$ - Process noise of model;

$V_{t c}=-\dot{D}$ - Target approach speed.

The state equation of the above model has the form:

$$
\left[\begin{array}{c}
\varepsilon_{d} \\
\omega_{d} \\
j_{t} \\
\dot{j}_{t}
\end{array}\right]=\left[\begin{array}{cccc}
0 & 1 & 0 & 0 \\
0 & \frac{2 V_{t c}}{D} & \frac{1}{D} & 0 \\
0 & 0 & 0 & 1 \\
0 & 0 & -\omega^{2} & 0
\end{array}\right]\left[\begin{array}{c}
\varepsilon_{d} \\
\omega_{d} \\
j_{t} \\
\dot{j}_{t}
\end{array}\right]+\left[\begin{array}{c}
0 \\
1 \\
-\frac{1}{D} \\
0 \\
0
\end{array}\right] j_{d}+\left[\begin{array}{c}
0 \\
0 \\
0 \\
\omega \xi_{t}
\end{array}\right]
$$

In the Weaving model, the level 2 integral stage is the forming filter. In order to create a sinusoidal maneuvering style with unchanged maneuvering amplitude and maneuver moment is evenly distributed in self-guidance time, the spectral density function of the process $\xi_{t}$ has the form: $\sigma_{a_{4}}^{2}=\omega^{2} \frac{j_{\max 4}^{2}}{T_{f}}$.

$j_{\max 4}$ - The maximum normal acceleration of the target can maneuver;

$T_{f}$ - Self-guidance time. $\omega$ - The maneuvering frequency of the target, considered to be known or within a certain range.

Discrete Weaving model, we have the following parameters: 
$\Phi_{m 4}=\left[\begin{array}{cccc}1 & \frac{D(\beta-1)}{2 V_{t c}} & \frac{D \omega(\beta-\cos X)-2 V_{t c} \sin X}{\omega\left(D^{2} \omega^{2}+4 V_{t c}^{2}\right)} & \frac{2 V_{t c} \cos X-D \omega \sin X}{\omega^{2}\left(D^{2} \omega^{2}+4 V_{t c}^{2}\right)}-\frac{1}{2 V_{t c} \omega^{2}}+\frac{D^{2} \beta}{2 V_{t c}\left(D^{2} \omega^{2}+4 V_{t c}^{2}\right)} \\ 0 & \beta & \frac{2 V_{t c}(\beta-\cos X)+D \omega \sin X}{D^{2} \omega^{2}+4 V_{t c}^{2}} & \frac{D \omega(\beta-\cos X)-2 V_{t c} \sin X}{\omega\left(D^{2} \omega^{2}+4 V_{t c}^{2}\right)} \\ 0 & 0 & \cos X & \frac{\sin X}{\omega} \\ 0 & 0 & -\omega \sin X & \cos X\end{array}\right]$

$\Phi_{m 4}$ - State transition matrix;

$G_{m 4}=\left[\begin{array}{c}\frac{T}{2 V_{t c}}+\frac{D(1-\beta)}{4 V_{t c}^{2}} \\ \frac{1-\beta}{2 V_{t c}} \\ 0 \\ 0\end{array}\right]$ - Control matrix.

Discrete form of the process noise covariance matrix of the Weaving model:

$$
\begin{aligned}
& Q_{k}^{\text {Weaving }}(1,1)=\left[T\left(D^{4} \omega^{4}+8 D^{2} V_{t c}^{2} \omega^{2}+16 V_{t c}^{4}\right)-(\beta-1)\left(\frac{D^{5} \omega^{4}}{V_{t c}}+4 V_{t c} D^{3} \omega^{2}\right)\right. \\
& -\sin X\left(8 \omega D^{2} V_{t c}^{2}+\frac{32 V_{t c}^{4}}{\omega}\right)+T\left(2 D^{2} V_{t c}^{2} \omega^{2}+8 V_{t c}^{4}\right)-(\cos X-1)\left(4 D^{3} V_{t c} \omega^{2}+16 D V_{t c}^{3}\right) \\
& +\cos X \sin X\left(\frac{8 V_{t c}^{4}}{\omega}-2 \omega D^{2} V_{t c}^{2}\right)+8 D V_{t c}^{3}\left(\cos ^{2} X-1\right)+\frac{D^{5} \omega^{4}}{4 V_{t c}}\left(\beta^{2}-1\right) \\
& \left.+4 D^{3} V_{t c} \omega^{2}(\beta \cos X-1)\right] /\left[4 D^{4} V_{t c}^{2} \omega^{8}+32 D^{2} V_{t c}^{4} \omega^{6}+64 V_{t c}^{6} \omega^{4}\right] \\
& Q_{k}^{\text {Weaving }}(1,2)=Q_{k}^{\text {Weaving }}(2,1)=\left[(1-\cos X)\left(2 \omega D^{2} V_{t c}+\frac{8 V_{t c}^{3}}{\omega}\right)+(1-\beta)\left(2 \omega D^{2} V_{t c}+\frac{D^{4} \omega^{3}}{2 V_{t c}}\right)\right. \\
& -\sin ^{2} X\left(\frac{4 V_{t c}^{3}}{\omega}-\omega D^{2} V_{t c}\right)+\sin X\left(D^{3} \omega^{2}+4 D V_{t c}^{2}\right)-4 D V_{t c}^{2} \cos X \sin X+\frac{D^{4} \omega^{3}}{4 V_{t c}}\left(\beta^{2}-1\right) \\
& \left.-D^{3} \omega^{2} \beta \sin X+2 D^{2} V_{t c} \omega(\beta \cos X-1)\right] /\left[2 D^{4} V_{t c} \omega^{7}+16 D^{2} V_{t c}^{3} \omega^{5}+32 V_{t c}^{5} \omega^{3}\right] \\
& Q_{k}^{\text {Weaving }}(1,3)=Q_{k}^{\text {Weaving }}(3,1)=\left[(\cos X-1)\left(\omega D^{2}+\frac{4 V_{t c}^{2}}{\omega}\right)-\frac{2 V_{t c}^{2}}{\omega}\left(\cos ^{2} X-1\right)+D V_{t c} \cos X \sin X\right. \\
& \left.-D V_{t c} \omega T-\frac{D^{4} \omega^{3}}{D^{2} \omega^{2}+4 V_{t c}^{2}}(\beta \cos X-1)+\frac{2 D^{3} V_{t c} \omega^{2} \beta \sin X}{D^{2} \omega^{2}+4 V_{t c}^{2}}\right] /\left[2 D^{2} V_{t c} \omega^{5}+8 V_{t c}^{3} \omega^{3}\right] \\
& Q_{k}^{\text {Weaving }}(1,4)=Q_{k}^{\text {Weaving }}(4,1)=\left[2 V_{t c}^{2} T-\sin X\left(D^{2} \omega+\frac{4 V_{t c}^{2}}{\omega}\right)+D V_{t c}\left(\cos ^{2} X-1\right)\right. \\
& \left.+\frac{2 V_{t c}^{2} \cos X \sin X}{\omega} \cdot \frac{D^{4} \omega^{3} \beta \sin X}{D^{2} \omega^{2}+4 V_{t c}^{2}}+\frac{2 D^{3} V_{t c} \omega^{2}}{D^{2} \omega^{2}+4 V_{t c}^{2}}(\beta \cos X-1)\right] /\left[2 D^{2} V_{t c} \omega^{4}+8 V_{t c}^{3} \omega^{2}\right] \\
& Q_{k}^{\text {Weaving }}(2,2)=\left[T\left(\frac{D^{2} \omega^{2}}{2}+2 V_{t c}^{2}\right)+\sin 2 X\left(\frac{D^{2} \omega}{4}-\frac{V_{t c}^{2}}{\omega}\right)-D V_{t c}(\cos 2 X-1)\right. \\
& \left.-2 D^{2} \omega \beta \sin X+\frac{D^{3} \omega^{2}}{4 V_{t c}}\left(\beta^{2}-1\right)\right] /\left[D^{4} \omega^{6}+8 D^{2} V_{t c}^{2} \omega^{4}+16 V_{t c}^{4} \omega^{2}\right]
\end{aligned}
$$




$$
\begin{aligned}
& Q_{k}^{\text {Weaving }}(2,3)=Q_{k}^{\text {Weaving }}(3,2)= {\left[\frac{D\left(\cos ^{2} X-1\right)}{2}-V_{t c} T+\frac{V_{t c} \cos X \sin X}{\omega}\right.} \\
&\left.-\frac{D^{3} \omega^{2}(\beta \cos X-1)}{D^{2} \omega^{2}+4 V_{t c}^{2}}+\frac{2 D^{2} V_{t c} \omega \beta \sin X}{D^{2} \omega^{2}+4 V_{t c}^{2}}\right] /\left[D^{2} \omega^{2}+4 V_{t c}^{2} \omega^{2}\right] \\
& Q_{k}^{\text {Weaving }}(2,4)=Q_{k}^{\text {Weaving }}(4,2)= {\left[\frac{V_{t c}\left(\cos ^{2} X-1\right)}{\omega}-\frac{D \omega T}{2}-\frac{D \cos X \sin X}{2}+\frac{D^{3} \omega^{2} \beta \sin X}{D^{2} \omega^{2}+4 V_{t c}^{2}}\right.} \\
&\left.+\frac{2 D^{2} V_{t c} \omega}{D^{2} \omega^{2}+4 V_{t c}^{2}}(\beta \cos X-1)\right] /\left[D^{2} \omega^{3}+4 V_{t c}^{2} \omega\right]
\end{aligned}
$$

$Q_{k}^{\text {Weaving }}(3,3)=\frac{2 X-\sin 2 X}{4 \omega^{3}}$

$Q_{k}^{\text {Weaving }}(3,4)=Q_{k}^{\text {Weaving }}(4,3)=\frac{1-\cos 2 X}{4 \omega^{2}}$

$Q_{k}^{\text {Weaving }}(4,4)=\frac{T}{2}+\frac{\sin 2 X}{4 \omega}$

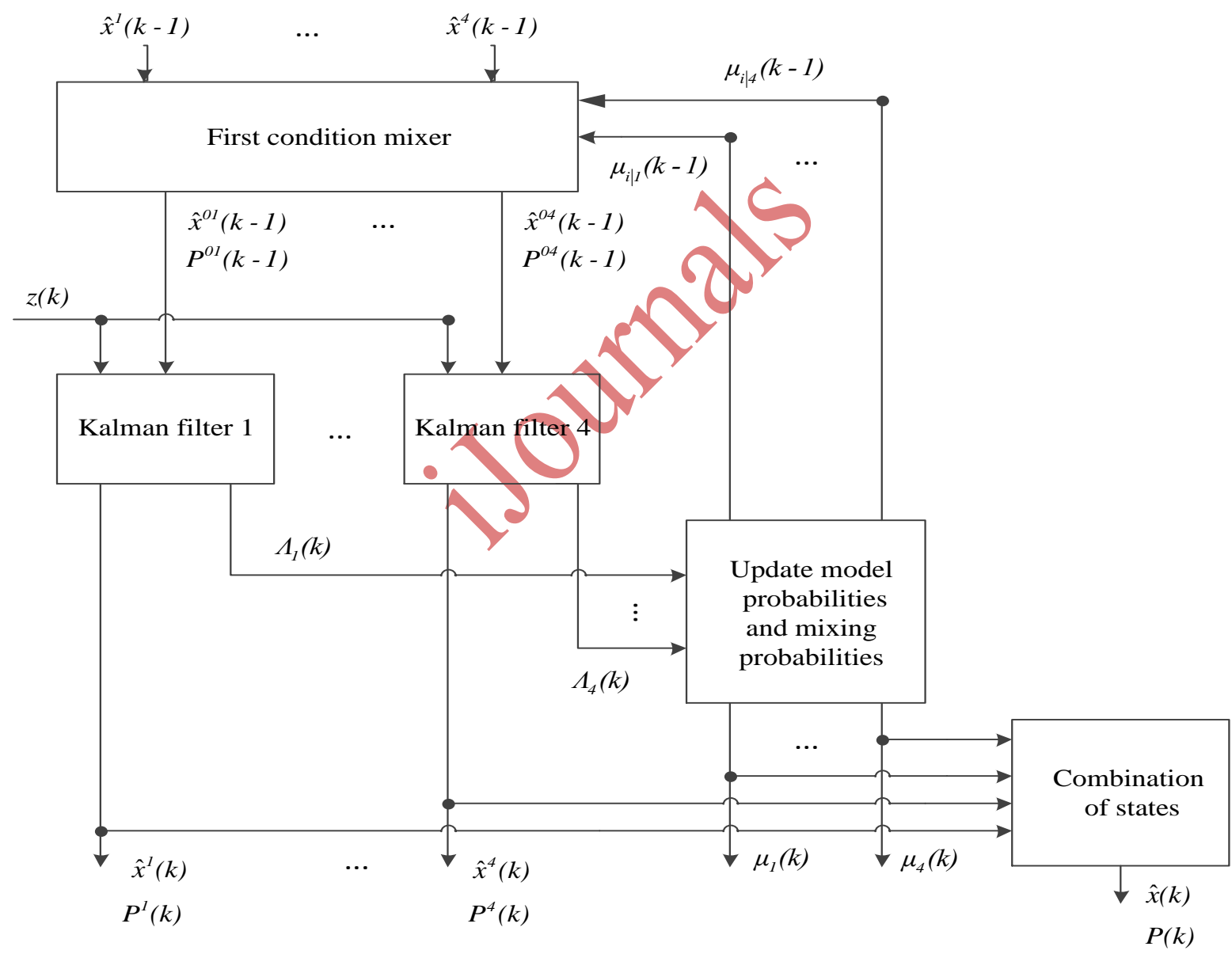

Figure 2. Block diagram of line of sight angle coordinate filter with $\mathbf{4}$ component Kalman filters

\section{SIMULATION RESULTS AND ANALYSIS}

The results of the simulation of the target angular coordinate system using the IMM4 algorithm with a snake style maneuver type with the initial parameters of the target and missile are as follows:
Inside: $\beta=e^{\frac{2 V_{V} T}{D}}, X=\omega T, T$ - Discrete cycle.

Thus, the line of sight angle coordinate filter is built on the basis of a interactive multi-model algorithm with 4 models: CV model, Singer model, CA model and Weaving model, I call it IMM4.
Update mode

$P(k)$
- The target's initial position: $x_{t}(0)=40(\mathrm{~km})$; $y_{t}(0)=O(\mathrm{~km})$.

- The missile's initial position: $x(0)=O(\mathrm{~km})$; $y(0)=O(\mathrm{~km})$.

- The target flies in at velocity: $350(\mathrm{~m} / \mathrm{s})$. 
- Missile velocity: $1000(\mathrm{~m} / \mathrm{s})$.

- The angle of the target's initial trajectory tilt: $\theta_{t}=0^{\circ}$.

- Normal target acceleration has the following form:

$j_{t}=30 \sin \left(\frac{1}{2} t\right)$

+ The maneuvering amplitude: $j_{\max }=30\left(\mathrm{~m} / \mathrm{s}^{2}\right)$

+ The maneuvering frequency: $\omega_{m}=0,5(\mathrm{rad} / \mathrm{s})$

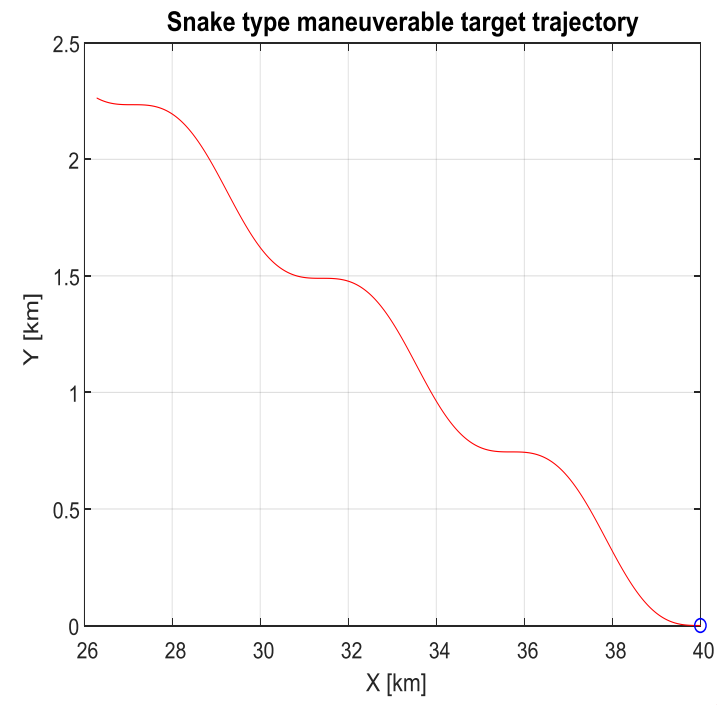

Figure 3. Snake type maneuverable target

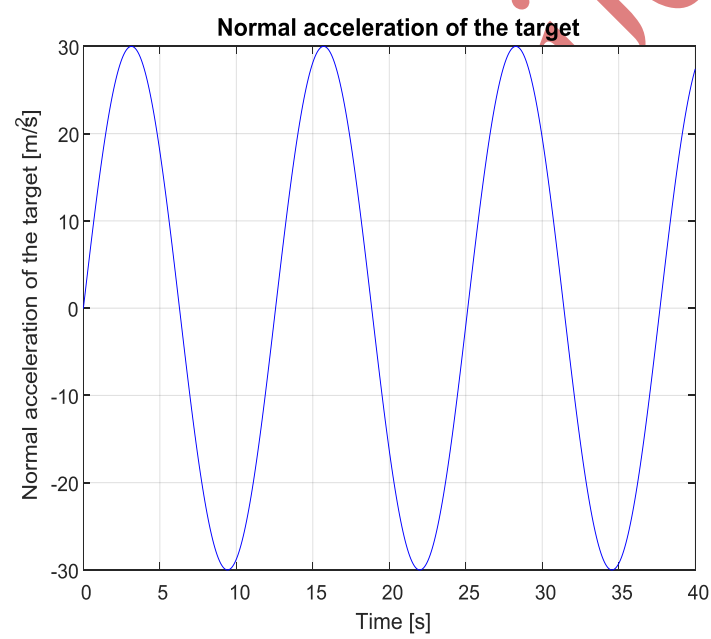

Figure 4. Normal acceleration of the target

From figures 3 and 4, we see, the target has an unforeseen form of maneuver, in which the timing of maneuver and the maneuver intensity is purely random.

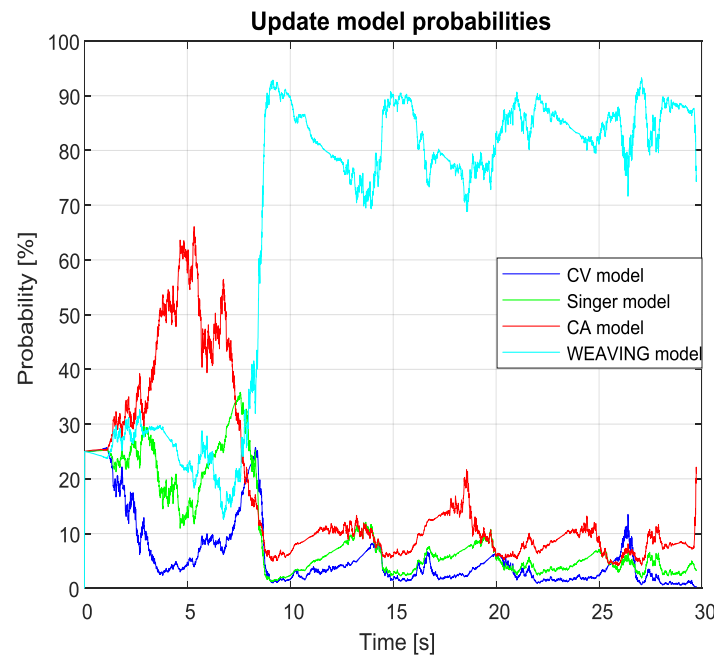

Figure 5. Graph of updating model probabilities of the IMM4 filter

Figure 5, shows that, after about 8 seconds, the IMM4 algorithm realizes the actual maneuver form of the target. This is reflected in the advantage of the snake style maneuvering model (Weaving model) over the rest model when updating the model probability. As a result, the target's phase coordinate evaluations will be significantly improved.

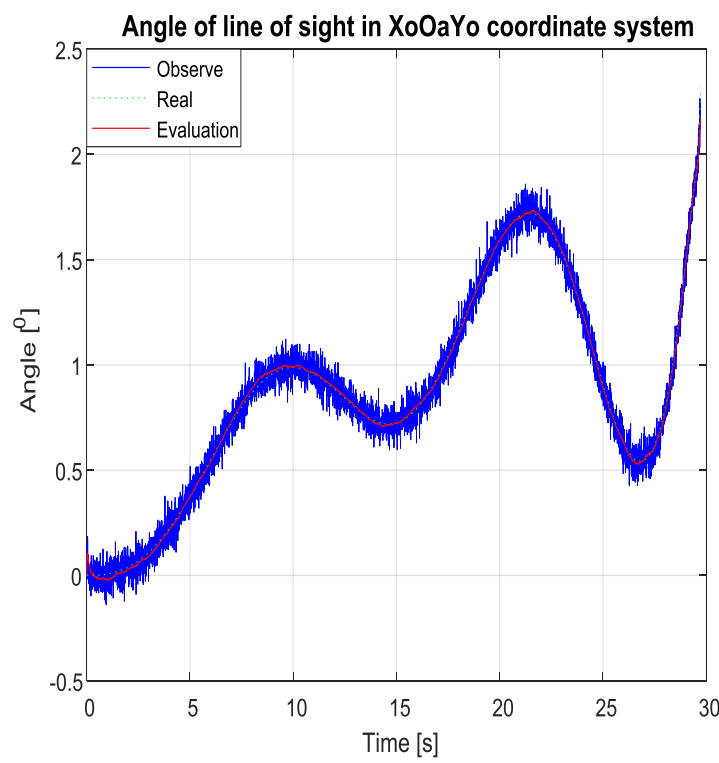

Figure 6. Evaluate the angle of line of sight of the IMM4 filter 


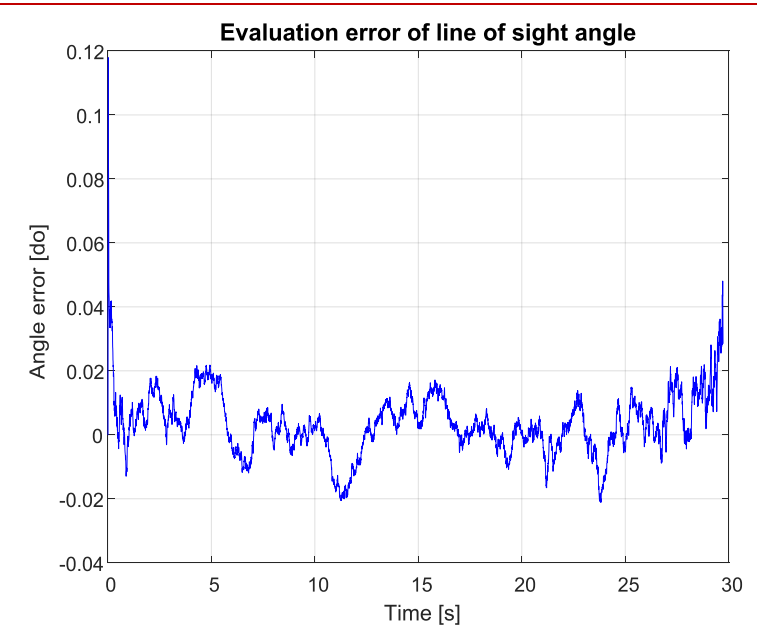

Figure 7. Evaluation error of the IMM4 filter

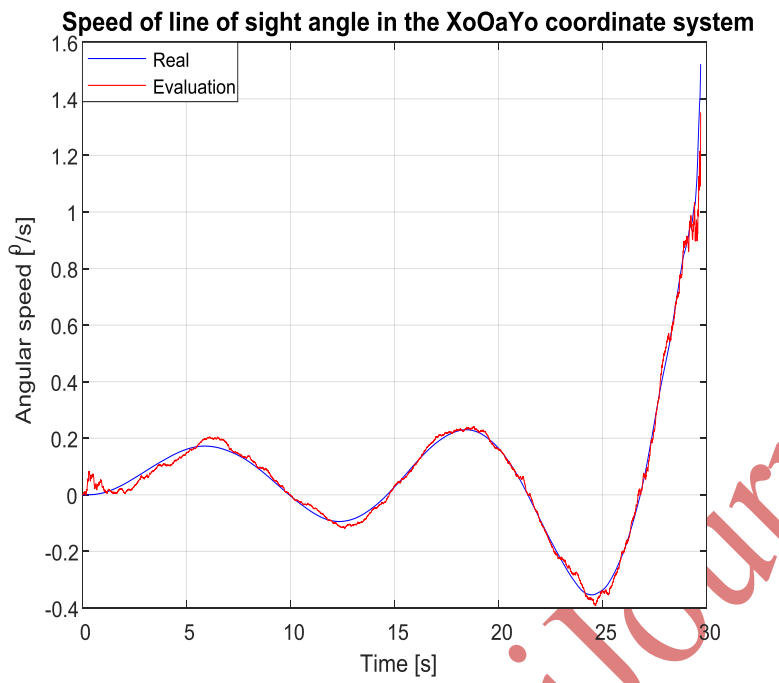

Figure 8. Speed evaluation of line of sight angle of the IMM4 filter

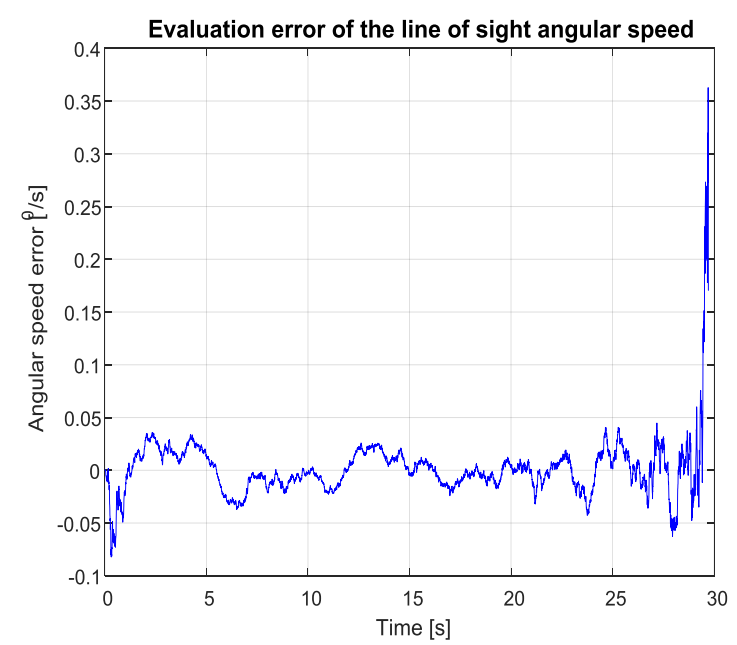

Figure 9. Evaluation error of the IMM4 filter

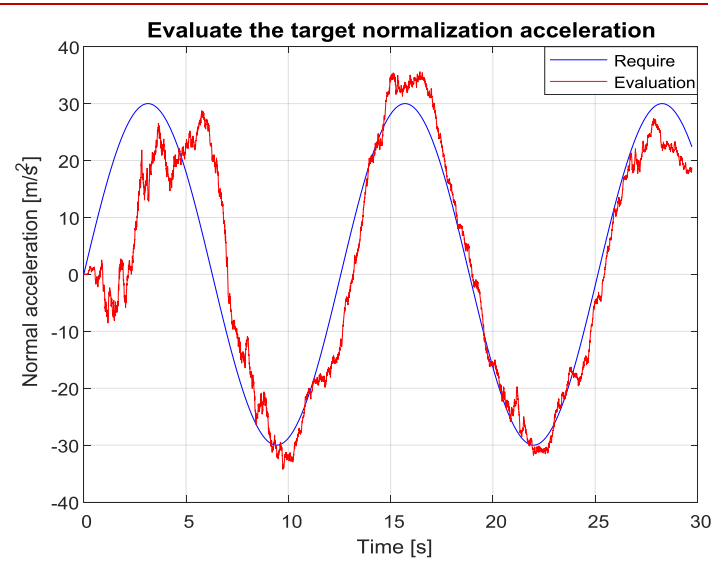

Figure 10. Evaluate the target normalization acceleration of the IMM4 filter

Calling IMM3 is a filter algorithm with 3 initial models (model CV, Singer, CA). We compare the quality of the line of sight angle coordinate filter using IMM3 filter algorithm with IMM4 filter algorithm after 100 Monte-Carlo runs:

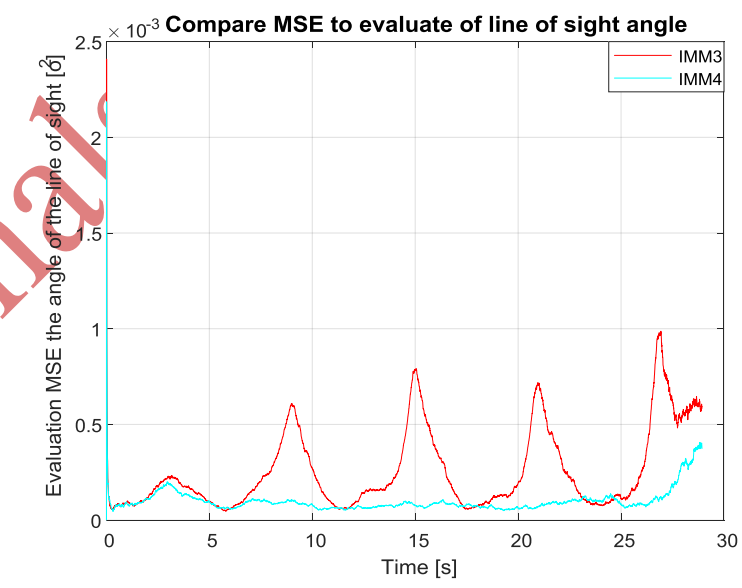

Figure 11. Compare MSE to evaluate of line of sight angle between IMM3 and IMM4 filtering algorithm

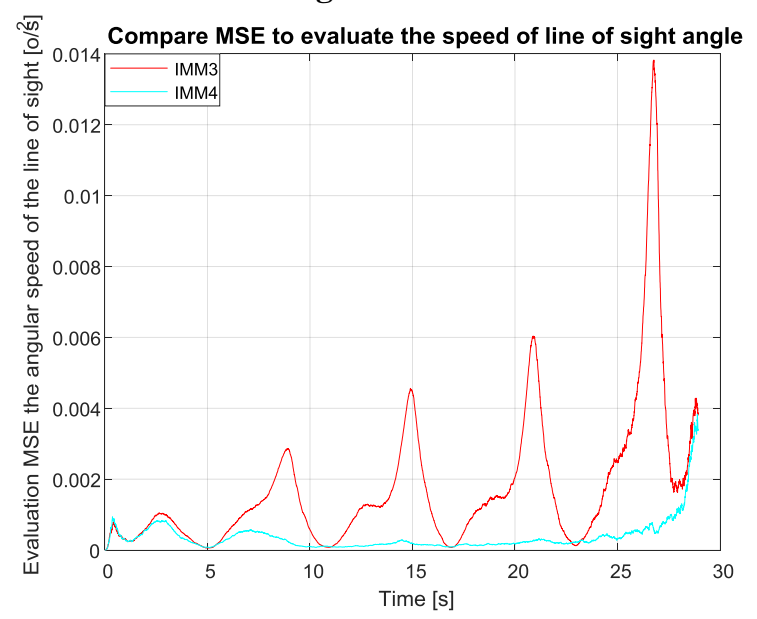

Figure 12. Compare MSE to evaluate the speed of line of sight angle between IMM3 and IMM4 filtering algorithm 
Volume 9 Issue 4 April 2021

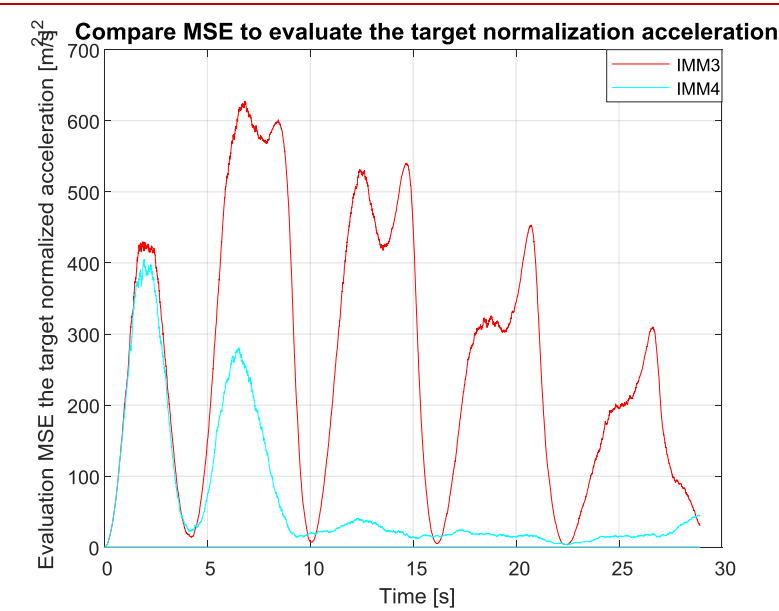

Figure 13. Compare MSE to evaluate the target normalization acceleration between IMM3 and IMM4 filtering algorithm

Results of running 100 times of Monte-Carlo shows that the IMM4 filter algorithm significantly improves the quality of the target phase coordinate filter when the evaluation error is reduced. When entering stable tracking (after 10 seconds) with line of sight angle $\operatorname{MSE}\left(\varepsilon_{d}\right) \approx 0,1 \times 10^{-3}\left[(o)^{2}\right]$, with angular speed of line of sight $\operatorname{MSE}\left(\omega_{d}\right) \approx 0,2 \times 10^{-3}\left[(\mathrm{o} / \mathrm{s})^{2}\right], \quad$ with target normalization $\operatorname{MSE}\left(j_{t}\right) \approx 20\left[\left(m / s^{2}\right)^{2}\right]$.

\section{CONCLUSION}

The maneuver of the target directly affect to the line of sight angle coordinate evaluation filter. So, to synthesize the target angle coordinate determination system with high accuracy in the maneuver and super maneuverable target conditions, just improve and advance the line of sight angle coordinates evaluation filter, the other filters are kept intact.

Surveying and evaluating the coordinate system of the target angle with the snake style maneuvering form (the popular maneuvering type in practice) increases the reliability of the algorithm when this model is included in the synthese process of the target phase coordinate filter. Of course, the more models that are taken into account when synthesizing the line of sight angle coordinate filter, the higher the adaptability of the filter to target maneuverability but will make the evaluation algorithm more complicated and increase computation mass and real time response for the electronics computer on board.

\section{REFERENCES}

[1] Li X.R., Jilkov V.P. Survey of maneuvering target tracking. IEEE transaction on aerospace and electronic systems, 41 (4) 2015, pp. 12551321.

[2] Xu Benlian. An adaptive tracking algorithm for bearings-only maneuvering target. International journal of computer science and network security, 7 (1) 2017.

[3] Канащенкова. А.И и Меркулова. В.И. Авиационные системы радиоуправления (том 2). Радиотехника, Москва, 2013.

[4] Wu N.E., Youmin Zhang, Kermin Zhou. Detection, estimation and accommodation of loss of control effectiveness. International journal of adaptive control and signal processing 14, 2010, pp. 775-795.

[5] Bar-Shalom Y., RongLi X., Kirubarajan T. Estimation with Applications to Tracking and Navigation. Theory Algorithms and Software, John Wiley \& Sons, 2011.

[6] Lee Jik Bum, Joo Young Hoon, Park Jin Bea. IMM method using intelligent input estimation for maneuvering target tracking. ICCAS 2013, pp. 1278-1282.

[7] Nguyen Van Bang. Synthesis of remote control law when taking into dynamics and nonlinear of the missile stage. Intelligent Systems and Networks (ICISN 2021, AISC 1386), Springer, No.22, DOI:10.1007/978-981-16-2094-2_22, April 12, 2021.

[8] Nguyen Van Bang, Hoang Van Ngoi. Synthesis of the maneuvering target coordinate determination algorithm on the basis of application of the interactive multi-model adaptive filtering technique. East European Scientific Journal, Vol 1, No.67, 3/2021, DOI: 10.31618/ESSA.2782-1994.2021.1.67, pp. 1623. 